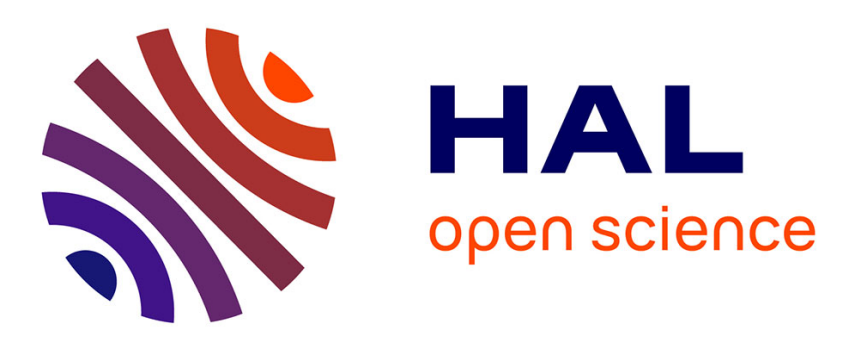

\title{
Design Procedure for a Fast and Accurate Parallel Manipulator
}

\author{
Sébastien Briot, Stéphane Caro, Coralie Germain
}

\section{To cite this version:}

Sébastien Briot, Stéphane Caro, Coralie Germain. Design Procedure for a Fast and Accurate Parallel Manipulator. Journal of Mechanisms and Robotics, 2017, 9 (6), pp.061012. 10.1115/1.4038009 . hal-01587975

\section{HAL Id: hal-01587975 https://hal.science/hal-01587975}

Submitted on 13 Oct 2017

HAL is a multi-disciplinary open access archive for the deposit and dissemination of scientific research documents, whether they are published or not. The documents may come from teaching and research institutions in France or abroad, or from public or private research centers.
L'archive ouverte pluridisciplinaire HAL, est destinée au dépôt et à la diffusion de documents scientifiques de niveau recherche, publiés ou non, émanant des établissements d'enseignement et de recherche français ou étrangers, des laboratoires publics ou privés. 


\title{
Design Procedure for a Fast and Accurate Parallel Manipulator
}

\author{
Sébastien Briot* \\ CNRS \\ Laboratoire des Sciences \\ du Numérique de Nantes (LS2N) \\ UMR CNRS 6004 \\ 44321 Nantes, France \\ Email: Sebastien.Briot@Is2n.fr
}

\author{
Stéphane Caro \\ CNRS \\ Laboratoire des Sciences \\ du Numérique de Nantes (LS2N) \\ UMR CNRS 6004 \\ 44321 Nantes, France \\ Email: Stephane.Caro@Is2n.fr
}

\author{
Coralie Germain \\ Agrocampus Ouest \\ 35042 Rennes, France \\ Email: coralie.germain@agrocampus-ouest.fr
}

This paper presents a design procedure for a twodegree of freedom translational parallel manipulator, named IRSBot-2. This design procedure aims at determining the optimal design parameters of the IRSBot-2 such that the robot can reach a velocity equal to $6 \mathrm{~m} / \mathrm{s}$, an acceleration up to $20 \mathrm{G}$ and a multi-directional repeatability up to $20 \mu \mathrm{m}$ throughout its operational workspace. Besides, contrary to its counterparts, the stiffness of the IRSBot-2 should be very high along the normal to the plane of motion of its movingplatform. A semi-industrial prototype of the IRSBot-2 has been realized based on the obtained optimum design parameters. This prototype and its main components are described in the paper. Its accuracy, repeatability, elasto-static performance, dynamic performance and elasto-dynamic performance have been measured and analyzed as well. It turns out the IRSBot-2 has globally reached the prescribed specifications and is a good candidate to perform very fast and accurate pick-and-place operations.

\section{Introduction}

Parallel robots are more and more attractive for highspeed pick-and-place operations due to their lightweight architecture and high stiffness [1]. However, high velocities and high accelerations may lead to some vibrations that may affect the robot accuracy and dynamic performance, thus discarding those robots to be used as high-speed parallel robots for special tasks requiring good accuracy and high accelerations such as the assembly of electronic components on

\footnotetext{
*Address all correspondence to this author.
}

printed circuit boards.

Several robot architectures with four degrees of freedom (dof) and generating Schönflies motions [2] for highspeed operations have been proposed in the past decades [1, 3-6]. However, four dof robots are not always necessary, especially for some simple operations requiring only two translational dof in order to move a part from a working area to another. Therefore, several robot architectures providing two translational dof motions have been synthesized in the literature such as the five-bar mechanism $[7,8]$, the paraplacer [9] and several mechanisms with additional kinematic chains used to constrain the platform rotations $[6,10,11]$.

It is noteworthy that the foregoing architectures are all planar, i.e., all their elements are constrained to move in the plane of motion. As a result, all their elements are subject to bending effects along the normal to the plane of motion. Therefore, in order for the mechanisms to be stiff enough along this direction, their bodies are usually bulky, leading to high inertia and to low acceleration capacities.

A two-dof spatial translational robot, named IRSBot-2, IRSBot-2 standing for "IRCCyN Spatial Robot with $\mathbf{2}$ dof", was introduced in [12]. It was shown that this robot architecture may have better performance in terms of mass in motion, stiffness and workspace size with respect to its serial and parallel manipulator counterparts. The IRSBot-2 has a spatial architecture and the distal parts of its legs are not subject to bending, but to tension, compression and torsion only. Consequently, its stiffness is increased and its total mass is reduced. In the same vein, the Par-2 robot composed of four legs was introduced in [13] as a mean to increase the stiffness of its mobile platform along the normal to its plane of mo- 


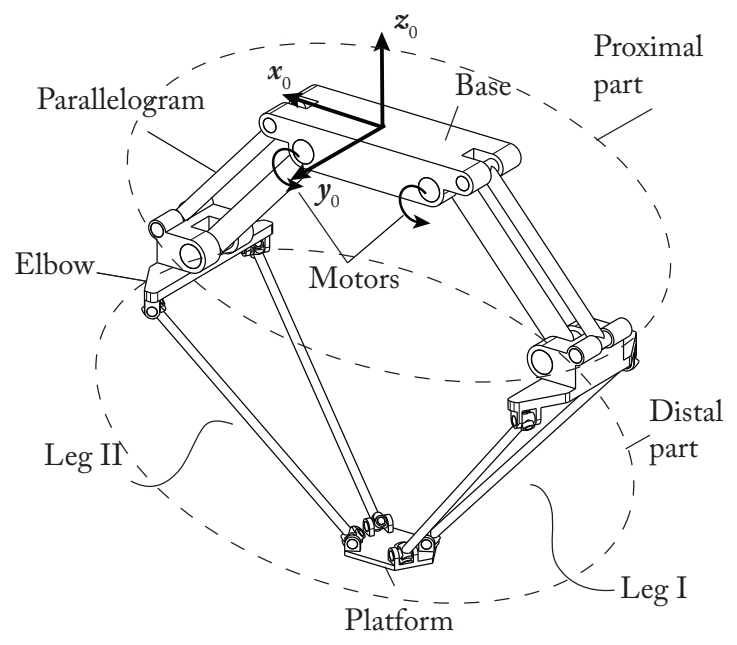

Fig. 1. CAD modeling of the IRSBot-2.

tion. However, contrary to the Par-2 robot, the IRSBot-2 is composed of two legs only in order to reduce the mechanism complexity and to increase its operational workspace size.

This paper introduces a design procedure for the IRSBot-2 such that the multi-directional repeatability and the dynamic performance of the robot are optimum. A running prototype, which was built based on the obtained design parameters, is described too. Some experimental validations were performed and are analyzed in this paper. Note that this paper is an improved version of [14] with the following new findings:

- on the multi-objective optimization problem (MOOP) for the design of the IRSBot-2: in [14], the three objective functions were normalized and weighted in order to convert the MOOP into a mono-objective optimization problem. Here, the Pareto-optimal solutions, i.e., the non-dominated solutions, of the MOOP at hand are presented.

- on the experimental validations of the design methodology: the IRSBot-2 semi-industrial prototype is described and its deflection, repeatability and dynamic performance are analyzed experimentally.

The paper is organized as follows. Section 2 presents the IRSBot-2 architecture and recalls its kinematic modeling, which is described into detail in [15]. The design procedure developed for the determination of the optimal design parameters of the IRSBot-2 is introduced in Section 3. A semi-industrial prototype of the IRSBot-2 and some experimental validations are described in Section 4. Finally, some conclusions are drawn in Section 5.

\section{Kinematics of the IRSBot-2}

\subsection{Robot architecture}

A CAD drawing of the IRSBot-2 architecture is shown in Fig. 1. The IRSBot-2 is a two-dof translational parallel manipulator. Its kinematic architecture is composed of two spatial limbs with identical joint arrangements.

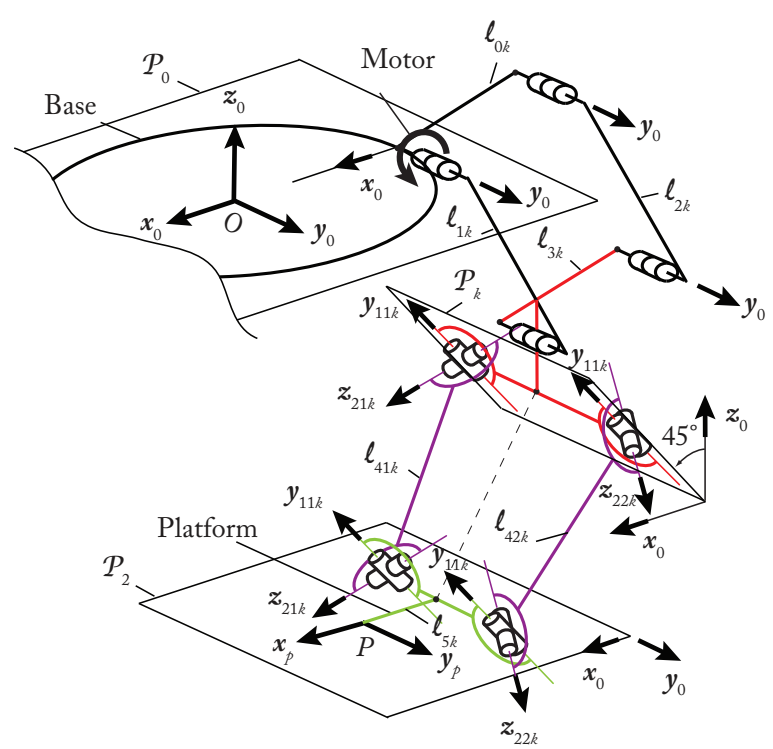

Fig. 2. Kinematic chain of the $k$ th leg $(k=I, I I)$.

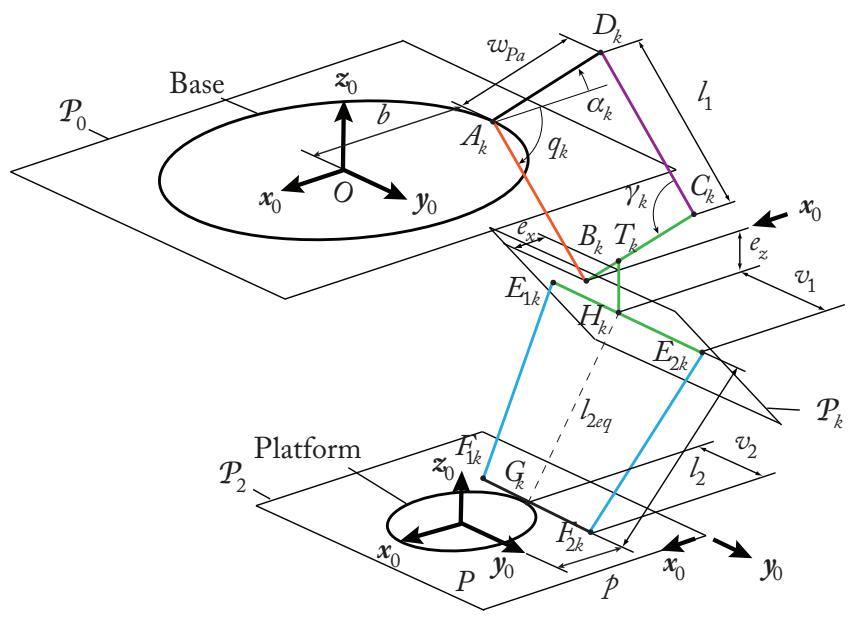

Fig. 3. Parametrization of the $k$ th leg $(k=I, I I)$.

The $k$ th leg of the IRSBot-2 is shown in Fig. 2. It is made of two modules: a proximal module and a distal module $(k=I, I I)$. Therefore, the robot is made of a proximal part and a distal part shown in Fig. 1. The proximal part is composed of the base and the two proximal modules. The distal part is composed of the moving-platform and the two distal modules. The base frame $\left(O, \boldsymbol{x}_{0}, \boldsymbol{y}_{0}, \boldsymbol{z}_{0}\right)$ is attached to plane $\mathcal{P}_{0}$.

The proximal module is a planar parallelogram, also named $\Pi$ joint, moving in the $\left(O, x_{0}, z_{0}\right)$ plane and is composed of links $\ell_{0 k}, \ell_{1 k}, \ell_{2 k}$ and $\ell_{3 k}$. The proximal module keeps the angle between planes $\mathcal{P}_{0}$ and $\mathcal{P}_{k}$ equal to $45 \mathrm{deg}$.

The distal module is linked to body $\ell_{3 k}$ of the parallelogram through two universal joints at points $E_{j k}$. The first axis of the universal joints $\boldsymbol{y}_{11 k}$ is located in planes $\mathcal{P}_{k}$ and $\left(\boldsymbol{x}_{0} E_{j k} \boldsymbol{y}_{0}\right)$. Moreover, the distal module is also linked to the 

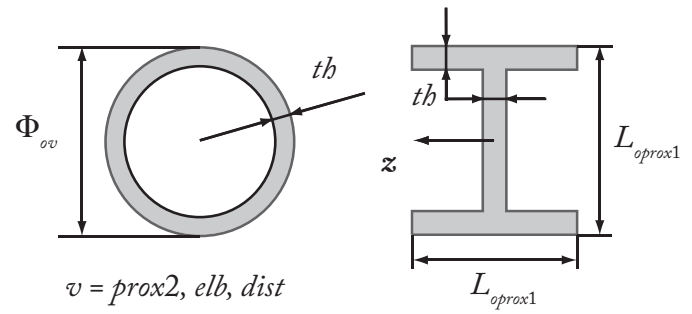

Fig. 4. Beam cross-section parameters

body $\ell_{5 k}$ of the moving platform through two universal joints located at points $F_{j k}(j=1,2)$. Axes $z_{21 k}$ and $z_{22 k}$ are symmetrical with respect to plane $\left(x_{0} O z_{0}\right)$. Links $\ell_{41 k}$ and $\ell_{42 k}$ are not parallel. This configuration prevents the distal module from becoming a spatial parallelogram and the robot architecture to be singular. The robot is assembled in such a way that the angle between planes $\mathcal{P}_{0}$ and $\mathcal{P}_{k}$ is equal to $45 \mathrm{deg}$. Therefore, plane $\mathcal{P}_{2}$ is parallel to plane $\mathcal{P}_{0}$.

The connection between the distal and proximal modules is made through the elbow, which is made up of segments $B_{k} T_{k}, T_{k} H_{k}$ and $E_{2 k} E_{1 k}$ as shown in Fig. 3.

\subsection{Singular configurations}

The singular configurations of the IRSBot- 2 were studied in [15]. The robot may reach three singularity types within its workspace, namely, Type 1 singularities [16] where the robot loses one dof, Type 2 singularities [16] where the platform gains a translational motion instantaneously as well as constraint singularities [17]. In the latter, the platform of the IRSBot-2 may gain a instantaneous rotational motion. The reader is referred to as [15] for a complete description of the IRSBot-2 singularities and to get the set of design parameters preventing the robot to reach any Type 2 and and constraint singularities within its operational workspace.

\subsection{Design parameters}

The design parameters of the IRSBot- 2 are shown in Fig. 3. For the $k$ th leg, $q_{k}$ is the actuated joint variable, $b=O A_{k}$ is the base radius, $l_{1}=A_{k} B_{k}$ is the length of the link $\ell_{2 k}, l_{2}=E_{j k} F_{j k}$ is the length of the links $\ell_{4 j k}, w_{P a}$ is the parallelogram width, $v_{1}$ and $v_{2}$ are the lengths of segments $E_{k} E_{j k}$ and $F_{k} F_{j k}$, respectively. The lenght $l_{2 e q}=H_{k} G_{k}$ is a constant equal to $l_{2 e q}=\sqrt{l_{2}^{2}-\left(v_{1}-v_{2}\right)^{2}}, v_{1}$ and $v_{2}$ being defined in Fig. 3. Finally, $\gamma_{k}=q_{k}+\alpha_{k}$ is the aperture angle of the parallelogram. $\alpha_{k}$ denotes the orientation angle of the link $\ell_{0 k}$ (Fig. 3). Position of point $T_{k}$ of the elbow is parameterized by variables $e_{x}$ and $e_{z}$ as shown in Fig. 3 .

In what remains, prox $_{1}$ denotes the actuated proximal arms $\ell_{1 k}$. prox $x_{2}$ denotes the passive proximal arms $\ell_{2 k}$. elb denotes the elbows $\ell_{3 k}$. dist denotes the distal arms $\ell_{4 j k}$. The bodies composing the proximal and distal modules, as well as the elbow, have hollow cylindrical cross-sections of outer diameter $\phi_{o v}$ and thickness $t h$, the subscript $v$ in $\phi_{o v}$ taking the value prox $_{1}$, prox $_{2}$, elb or dist, except for the body prox $_{1}$ which is made of an I-shape beam leading to a better behavior when faced with bending effects (Fig. 4).

In order to reduce the number of design variables and to simplify the design problem the beams are supposed to have the same thickness $t h$. Similarly, the height and width of the I-shape beams are the same and equal to $L_{\text {oprox } 1}$. Moreover, the moving platform is considered as rigid.

As a consequence, the design parameters of the IRSBot2 are classified as follows:

Lengths: $l_{1}, l_{2}$ (or $\left.l_{2 e q}\right), b, p, w_{P a}, e_{x}, e_{z}, v_{1}$ and $v_{2}$;

Angles : $\alpha_{k}$;

Cross-section parameters : $\phi_{o v}, L_{o p r o x 1}, t h$.

Additionnally, one must consider the material parameters (Young and shear moduli, material density) that are assumed to have a known constant value.

\section{Optimal design procedure}

The design procedure developed to obtain the optimal parameters of the IRSBot-2 based on high-speed and accuracy requirements is described thereafter.

\subsection{Specifications}

The design specifications for IRSBot- 2 are summurized in Tab. $1^{1}$. The robot footprint should be as small as possible. Moreover, in order to reduce vibratory phenomena due to high inertial effects, the robot natural frequencies should be a maximum.

Table 2 gives the characteristics of the direct-drive motors chosen for designing the robots ${ }^{2}: V_{\max }$ is the maximal motor speed; $T_{\text {peak }}$ is the peak torque; $T_{C}$ is the rated torque; $J$ is the rotor inertia; $r$ is the motor encoder resolution. Moreover, the motor footprint is represented by a cylinder of diameter $\Phi$.

The global dimensions of the standard Adept pick-andplace cycle that the robot should perform within $200 \mathrm{~ms}$ are given in Tab. 1. Nevertheless, the trajectory is not strictly defined. A test trajectory is optimized to minimize the cycle time and to ensure that the moving platform acceleration remains lower than $20 \mathrm{G}$. The procedure to optimize this trajectory is given in Appendix. This test trajectory is used in the optimization process to verify that the robot fulfills the prescribed dynamic performance in terms of input torques.

\subsection{Optimal design problem formulation}

The design optimization process aims at determining the optimal values of the fifteen design parameters of the IRSBot-2 given in Sec. 2.1 based on geometric, kinematic, kinetostatic, elastic and dynamic performance.

It appears that the geometric, kinematic, kinetostatic performance of the robot depend on seven parameters only. Those parameters are grouped into

\footnotetext{
${ }^{1}$ These requirements were defined with industrial partners in the scope of the French project ARROW (ANR 2011 BS3 006 01).

${ }^{2}$ The motors were imposed by a project partner and are TMB 210150 ETEL motors http: //www. etel.ch/torque_motors/TMB
} 
Table 1. Specifications for the IRSBot-2

\begin{tabular}{ll}
\hline Repeatability $\varepsilon_{l i m}$ & $20 \mu \mathrm{m}$ \\
$\begin{array}{l}\text { End-effector resolution } r_{l i m} \\
\text { Maximum acceleration }\end{array}$ & $2 \mu \mathrm{m}$ \\
Cycle time & $20 \mathrm{G}$ \\
Path & $25 \mathrm{~mm} \times 300 \mathrm{~mm} \times 25 \mathrm{~mm}$ \\
Regular workspace size & $800 \mathrm{~mm} \times 100 \mathrm{~mm}$ \\
$\begin{array}{l}\text { Deformation } \delta_{t l i m} \text { under a } \\
\text { force } \mathbf{f}_{s}=[0,20,0] \mathrm{N} \text { and a } \\
\text { moment } \\
\mathbf{m}_{s}=[0.1,0.1,0.1] \mathrm{N} . \mathrm{m}\end{array}$ & {$[0.2,0.2,0.2] \mathrm{mm}$,} \\
& {$[0.2,0.2,0.2] \mathrm{deg}$} \\
$\begin{array}{l}\text { Maximum payload } \\
\text { (including the platform mass } \\
\text { and the gripper) }\end{array}$ & $1.5 \mathrm{~kg}$ \\
\hline
\end{tabular}

Table 2. Datasheet of the TMB210-150 ETEL motor

\begin{tabular}{cccccc}
\hline \hline $\begin{array}{c}V_{\max } \\
{[\mathrm{rpm}]}\end{array}$ & $r$ & $T_{\text {peak }}$ & $T_{C}$ & $\Phi$ & $J$ \\
\hline 600 & $280000 \times 4$ & 672 & 140 & 230 & $4.5 e^{-2}$ \\
\hline \hline
\end{tabular}

the vector $\mathbf{x}_{1}=\left[l_{1} l_{2 e q} \quad b \quad p \quad e_{x} e_{z} \alpha_{I}\right]$. The other parameters which are grouped into the vector $\mathbf{x}_{2}=$ $\left[\begin{array}{llll}v_{1} & v_{2} & w_{P a} & L_{\text {prox }_{1}}\end{array} \Phi_{\text {oprox }_{2}} \Phi_{\text {odist }} \Phi_{\text {oelb }} t h\right]$ affects the robot elastostatic, dynamic and elastodynamic performance only.

As the objective function and the constraints of the first optimization problem do not depend on vector $\mathbf{x}_{2}$, it is possible to formulate two design optimization problems. $\mathbf{x}_{1}$ is the decision variable vectors of the first problem while $\mathbf{x}_{2}$ is the decision variable vectors of the second problem. As a consequence, the two optimization problems can be solved in cascade by considering the optimal set of decision variables for the first optimization problem as constant parameters in the second optimization problem.

\subsubsection{First design problem formulation}

The first design optimization problem aims at finding the optimal decision variable vector $\mathbf{x}_{1}$ based on geometric, kinematic and kinetostatic performance.

Objective Function The objective function of the first optimization problem is the area $\mathcal{A}_{b b}$ of the surface area of the bounding rectangle (orange rectangle) shown in Fig. 5

The area $\mathcal{A}_{b b}$ is computed for the robot home configuration shown in Fig. 6. Therefore, $\mathcal{A}_{b b}$ is given by,

$$
\mathcal{A}_{b b}=b b_{l} b b_{h}
$$

$b b_{l}$ and $b b_{h}$ are the length and the height of the bounding rectangle.

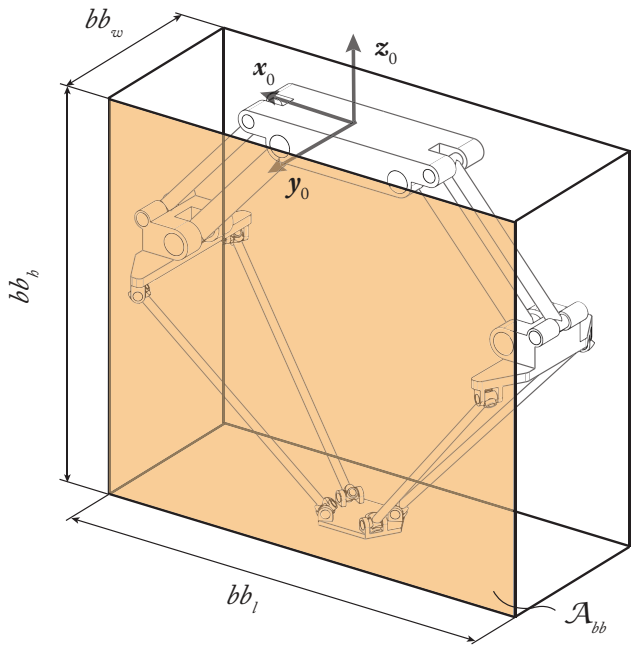

Fig. 5. Bounding box of the IRSBot-2.

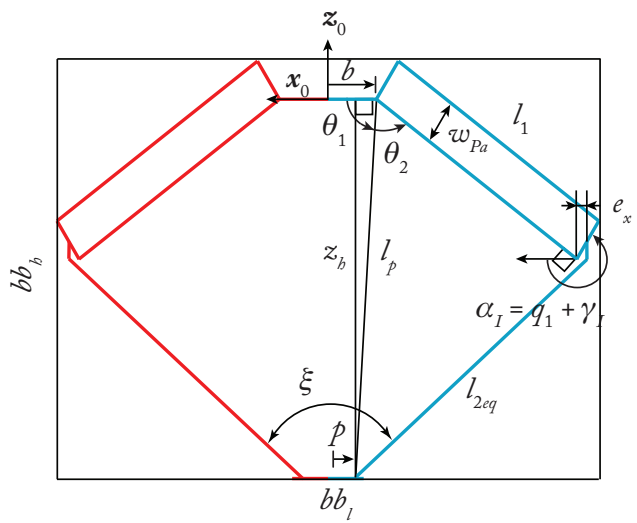

Fig. 6. Home configuration of the IRSBot-2.

Optimization Problem Formulation From Tab. 1, the IRSBot-2 workspace should be a rectangle, named Regular Workspace $R W$, of length $w_{l}=800 \mathrm{~mm}$ and height $w_{h}=100 \mathrm{~mm}$. Some geometric, kinematic and kinetostatic constraints should be also guaranteed within $R W$, thus defining the Regular Dexterous Workspace $(R D W)$ with specifications given in Tab. 1. Let $L R D W$ denote the Largest Regular Dexterous Workspace of the manipulator.

The design problem aims at finding the decision variable vector $\mathbf{x}_{1}$ that minimizes the surface area $\mathcal{A}_{b b}$ while the length $l_{L R D W}$ and the height $h_{L R D W}$ of $L R D W$ are higher or equal than $w_{l}$ and $w_{h}$, respectively. As a constraint, one should note that the base radius $b$ of the robot should also be greater than the motor radius $\Phi / 2$ given in Tab. 2. For design constraints,

- The base radius $b$ should be bigger than the moving platform radius $p$, which has been fixed to $50 \mathrm{~mm}$.

- The parameter $e_{z}$ is set to zero.

Thus, the design optimization problem is formulated as fol- 
lows,

$$
\begin{aligned}
& \text { minimize } \mathcal{A}_{b b}
\end{aligned}
$$

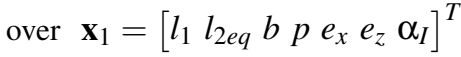

$$
\begin{aligned}
& \text { subject to } l_{L R D W} \geq w_{l}, h_{L R D W} \geq w_{h} \\
& b \geq p, b>\Phi / 2 \\
& p=50 \mathrm{~mm}, e_{z}=0 \mathrm{~mm}
\end{aligned}
$$

The methodology used to find $L R D W$ for a given vector $\mathbf{x}_{1}$ is explained below.

Largest Regular Dexterous Workspace The following geometric and kinematic constraints should be respected within $R W$ for the regular workspace to become dextrous:

1. $R W$ must be free of singularity [15];

2. The following constraints are fixed so that the degeneracy of the $\Pi$ joints is avoided:

$$
\begin{aligned}
\pi / 6 & \leq \gamma_{I} \leq 5 \pi / 6 \\
\pi+\pi / 6 & \leq \gamma_{I I} \leq \pi+5 \pi / 6
\end{aligned}
$$

where $\gamma_{k}=\alpha_{k}-q_{k}, k=I, I I$.

3. Quality of the velocity transmission: Based on the results of the definition of the optimal trajectory (see Appendix), the IRSBot-2 should be able to reach a velocity greater than $v^{\text {lim }}=6 \mathrm{~m} \cdot \mathrm{s}^{-1}$ in any point of $R W$. Knowing the maximal motor speed $V_{\max }$ (Tab. 2) and the robot Jacobian matrix $\mathbf{J}$ from [12], the minimal platform velocity $\dot{p}^{\min }$ at any point of $R W$ can be computed [18]. The following constraint should be satisfied throughout $R W$ :

$$
\dot{p}^{\text {min }}>v^{\lim }
$$

4. Error transmission: For a resolution $r$ of the motor encoders (Tab. 2), the maximal platform resolution $\delta p^{\max }$ can be computed using the first-order geometric model approximation [19]. Therefore, $\delta p^{\max }$ should be smaller than $\varepsilon_{\text {lim }}$ at any point of $R W, \varepsilon_{\text {lim }}$ being given in Tab. 1 .

5. The reaction forces into the passive joints are proportional to $1 / \sin \xi$ [20], where $\xi$ is the angle between the distal modules (Fig. 6). It is assumed that $\sin \xi$ should be bigger than 0.1 to avoid excessive efforts into the joints.

The algorithm given in [18] is used to find the LRDW amongst the RDWs of the manipulator for a given decision variable vector $\mathbf{x}_{1}$.

Optimal Solution of Problem (2) The ga MATLAB function was used to find an approximate solution to problem (2). Convergence was obtained after six generations with a population containing 150 individuals. Then, the MATLAB fmincon function was run to obtain a local optimum $\mathbf{x}_{1}^{*}$, taking the best individual of the final population as the starting point.

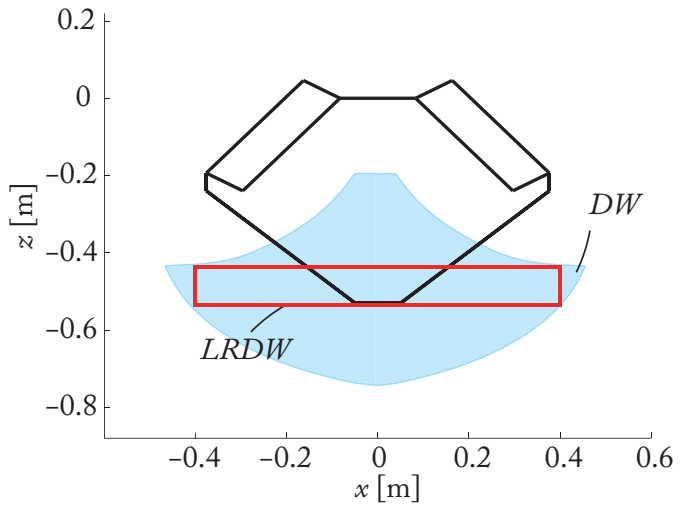

Fig. 7. Optimal 2D-design of the IRSBot-2 (solution to Pb. (2)) and largest regular dexterous workspace (scaled)

Table 3. Optimal solution of $\mathrm{Pb}$. (2)

\begin{tabular}{c|cccc}
\hline \hline $\mathcal{A}_{b b}\left[\mathrm{~m}^{2}\right]$ & $l_{1}[\mathrm{~mm}]$ & $l_{2 e q}[\mathrm{~mm}]$ & $b[\mathrm{~mm}]$ & $e_{x}[\mathrm{~mm}]$ \\
\hline 0.2226 & 321 & 437 & 83 & 80 \\
\hline \hline & $\alpha_{I}[\mathrm{deg}]$ & $e_{z}[\mathrm{~mm}]$ & $p[\mathrm{~mm}]$ & \\
\cline { 2 - 4 } & 210 & 0 & 50 \\
\end{tabular}

The optimal design variables of problem (2) and the associated surface area $\mathcal{A}_{b b}$ are given in Tab. 3 . The corresponding dimensions of the IRSBot- 2 and LRDW are depicted in Fig. 7.

\subsubsection{Second design optimization problem}

The second design optimization problem aims at finding the design variable vector $\mathbf{x}_{2}$ based on the prescribed dynamic and elastic performance of the IRSBot- 2 .

The elastostatic model and a dynamic model of the IRSBot-2 are expressed in [12,21]. An elastodynamic model of the robot was formulated based on the methodology presented in [22].

It was decided that the links of the IRSBot-2 would be made up of Duraluminum of Young modulus $E=74 \mathrm{MPa}$, shear modulus $G=27.8 \mathrm{MPa}$ and density $\rho=2800 \mathrm{Kg} \cdot \mathrm{m}^{-3}$. The shape of the links is parameterized in Fig. 4.

Three Objective Functions The optimization problem at hand has three objective functions. The first objective function is the width $b b_{w}$ of the bounding box (Fig. 5).The second objective function is the mass $M_{I R S}$ in motion of the manipulator, which depends on the link cross-sections and lengths. It should be noted that the mass of the platform $M_{\text {platform }}$ is a maximum and equal to $1.5 \mathrm{~kg}$, i.e. the value given in Tab. 1. The mass of the other links is computed by knowing the material density, the link length and cross-section.

Let $f_{I R S}^{1}$ be the smallest frequency from the natural frequencies computed at both ends of the optimal trajectory thanks to the aforementioned elastodynamic model. $f_{I R S}^{1}$ is the third objective function of the optimization problem. 
Constraints Constraints related to the elastostatic and dynamic performance of the robot are set. First, the robot input torques should be lower than the peak torque $T_{\text {peak }}$ (Tab. 2) along the test trajectory given in Appendix. Then, the rootmean-square $\tau_{R M S}$ of the motor torques should be smaller than the rated torque $T_{C}=140 \mathrm{Nm}$ in order to avoid motor over-heating.

Moreover, for a $20 \mathrm{~N}$ force applied along $\boldsymbol{y}_{0}$ on the robot platform, the displacement of the end-effector should be smaller than $0.2 \mathrm{~mm}$ whereever in the workspace. For a $0.1 \mathrm{Nm}$ moment applied on the robot platform about any axis, the orientation displacement of the end-effector should be smaller than $0.2 \mathrm{deg}$ whereever in the workspace. These constraints are expressed as $\delta_{t}^{\max } \leq \delta_{\text {tlim }}$ in the optimization problem formulation.

Optimization Problem Formulation The second design optimization problem can be formulated as follows,

$$
\begin{aligned}
\operatorname{minimize} & b b_{w} \\
\text { minimize } & M_{I R S} \\
\text { maximize } & f_{I R S}^{1} \\
\text { over } & \mathbf{x}_{2}=\left[\begin{array}{llll}
v_{1} & v_{2} w_{P a} & L_{\text {prox }_{1}} \Phi_{\text {oprox }_{2}} \Phi_{\text {odist }} \Phi_{\text {oelb }} \text { th }
\end{array}\right] \\
\text { subject to } & \tau_{\text {max }} \leq T_{\text {Peak }} \\
& \tau_{R M S} \leq T_{C} \\
& \delta_{t}^{\text {max }} \leq \delta_{\text {tlim }}
\end{aligned}
$$

Pareto-optimal solutions of Problem (5) The Pareto front represents the non-dominated solutions, also named Paretooptimal solutions, of the multi-objective optimization problem. It is obtained by using the evolutionary algorithm NSGA-II [23]. This algorithm is based on an evolutionary algorithm allowing the sorting of the non-domintaed solutions. This algorithm is known for its low complexity $O\left(M N^{2}\right), M$ being the number of objective functions and $N$ the population size, with respect to other multi-objective evolutionary algorithms, their complexity being usually equal to $O\left(M N^{3}\right)$.

The Pareto front obtained for problem (5) is shown in Fig. 8. Each blue circle corresponds to a non-dominated solution. It should be noted that the depicted population does not only correspond to the population obtained at the last generation of the algorithm. In order to obtain a larger design space, the depicted population contains the best individuals for each generated population.

Figure 9 illustrates the boundaries of the performance function space by the visualization of the associated design space. The design space is represented by a scaled drawing of the IRSBot- 2 robot. The associated objectives are summed up in Tab. 4.

Let us consider a reference solution $\mathbf{s}^{\star}$, which belongs to the set of Pareto-optimal solutions. This reference solution is the lightest one amongst all Pareto-optimal solutions. Its first natural frequency $f_{I R S}^{1}\left(\mathbf{s}^{\star}\right)$ is equal to $49 \mathrm{~Hz}$. The solution $\mathbf{s}^{\star}$ is shown in black on the Pareto front as shown in Fig. 8. Figure 10 and Table 5 illustrate and sum up the design parameters associated to this solution, while Tab. 6 sums up the ob-

\begin{tabular}{|c|c|c|c|}
\hline Design & $b b_{w}[\mathrm{~m}]$ & $M_{I R S}[\mathrm{~kg}]$ & $f_{I R S}^{1}[\mathrm{~Hz}]$ \\
\hline Yellow ( ) & 0.20 & 1.94 & 41.9 \\
\hline Pink $(+)$ & 0.15 & 2.03 & 40.5 \\
\hline Orange $(\star)$ & 0.15 & 2.19 & 43.9 \\
\hline Purple ( ) & 0.15 & 2.28 & 46.2 \\
\hline Green (ם) & 0.2 & 2.19 & 49.4 \\
\hline Blue $(\boldsymbol{\Delta})$ & 0.23 & 2.07 & 46.9 \\
\hline
\end{tabular}
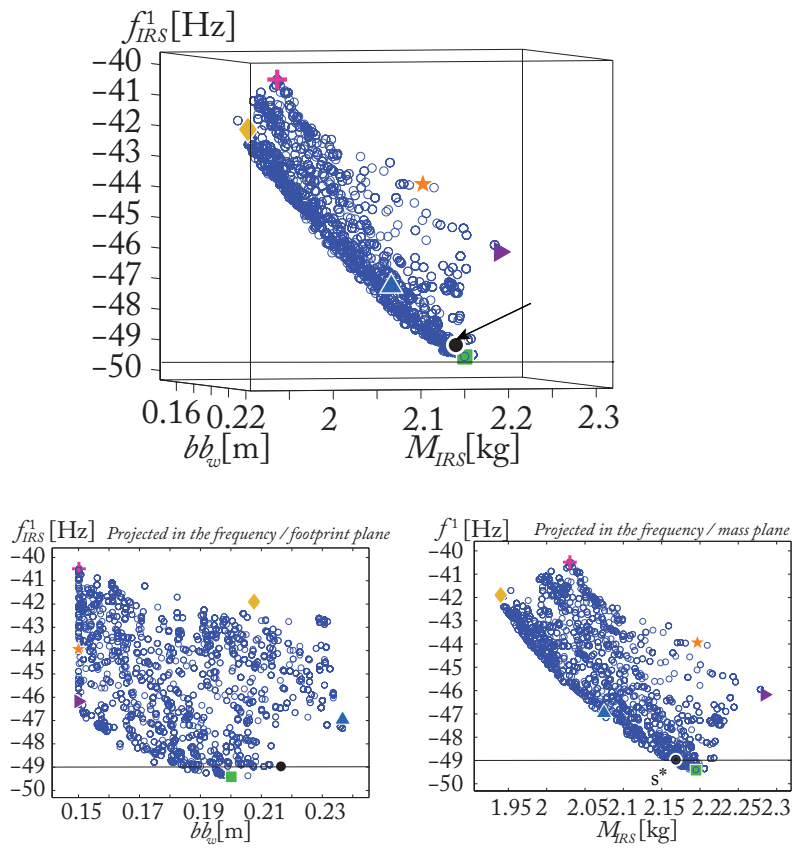

Fig. 8. Pareto front of the IRSBot-2

Table 4. Values of the objective functions for the extremal designs depicted in Figure 9

Table 5. Design variables for the optimal solution $\mathbf{s}^{\star}$

\begin{tabular}{cccccccc}
\hline \hline$v_{1}$ & $v_{2}$ & $w_{P a}$ & $L_{\text {prox }_{1}}$ & $\Phi_{\text {oprox }_{2}}$ & $\Phi_{\text {odist }}$ & $\Phi_{\text {oelb }}$ & th \\
\hline$[\mathrm{mm}]$ & {$[\mathrm{mm}]$} & {$[\mathrm{mm}]$} & {$[\mathrm{mm}]$} & {$[\mathrm{mm}]$} & {$[\mathrm{mm}]$} & {$[\mathrm{mm}]$} & {$[\mathrm{mm}]$} \\
216.4 & 50 & 93 & 91.2 & 10 & 39.7 & 50.9 & 2.3 \\
\hline \hline
\end{tabular}

jectives and the constraints corresponding to this design solution. $\delta_{t x}, \delta_{t y}, \delta_{t z}$ denote the translational point-displacements of the end-effector along $\boldsymbol{x}_{0}, \boldsymbol{y}_{0}$ and $z_{0}$, respectively. $\delta_{r x}, \delta_{r y}$, $\delta_{r z}$ represent the rotational displacement of the end-effector about axes $\boldsymbol{x}_{0}, \boldsymbol{y}_{0}$ and $\boldsymbol{z}_{0}$, respectively.

Solution $\mathbf{s}^{\star}$ has been selected to define the dimensions of the IRSBot-2 prototype, which is described thereafter.

\section{IRSBot-2 prototype}

This section describes the semi-industrial prototype of the IRSBot-2 and presents its performance that have been assessed experimentally. 


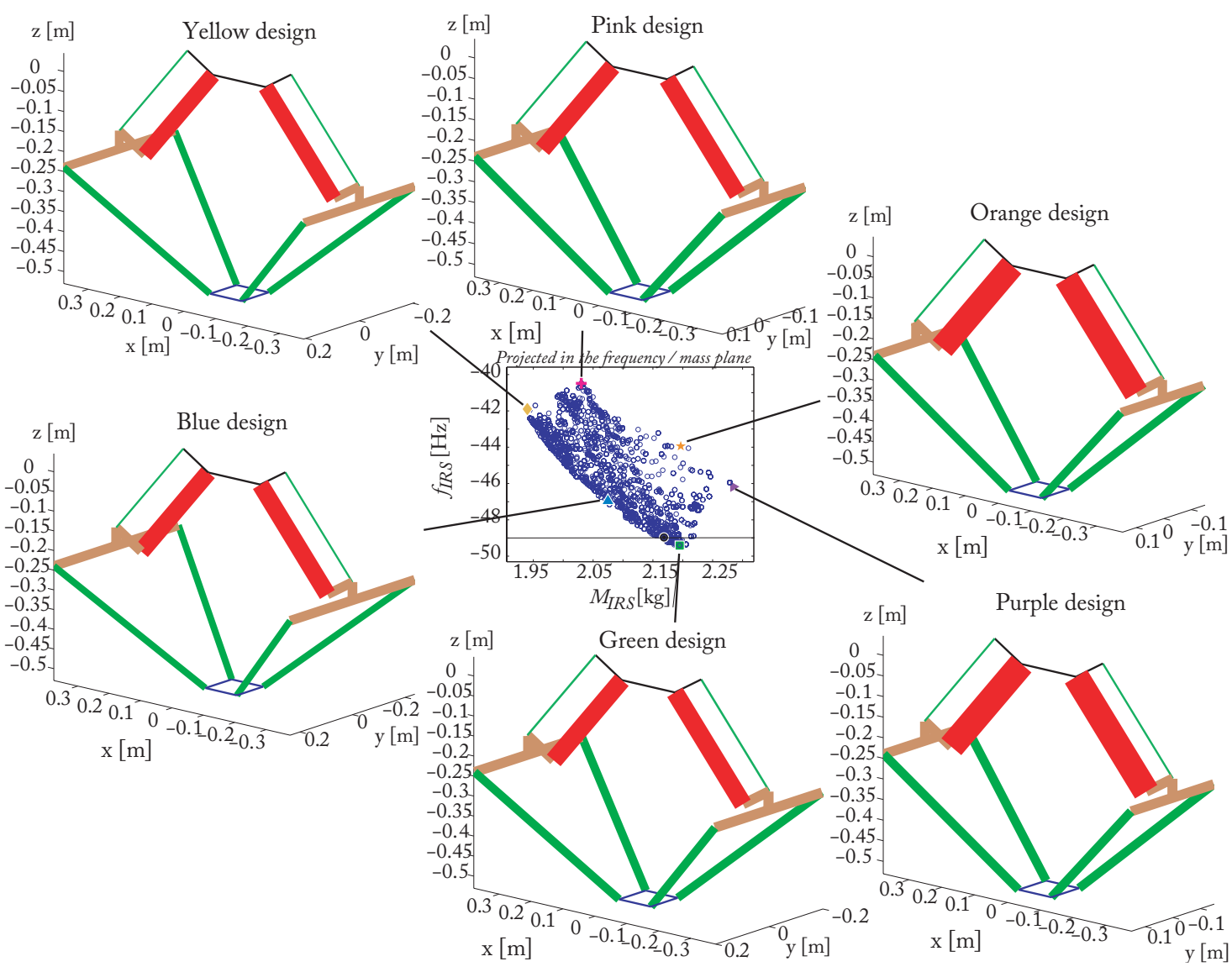

Fig. 9. Pareto-optimal solutions on the performance function space boundaries
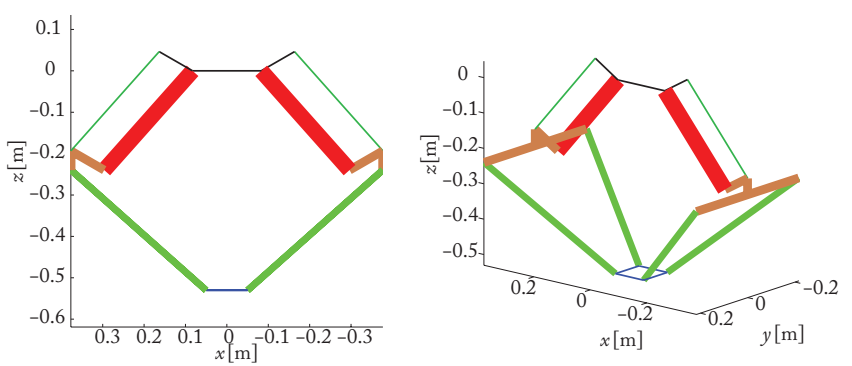

Fig. 10. Scaled representation of the optimal design solution $\mathbf{S}^{\star}$ for the IRSBot-2

\subsection{IRSBot-2 prototype performance}

A prototype of the IRSBot- 2 has been realized based on the foregoing results and is shown in Fig. 11. Details on its key components can be found in [24], especially the design of joints with low clearance and high stiffness.

To make the robot move, the motor amplifiers receive a command that is proportional to the actuator torques. The prototype is equipped with a dSPACE 1103 control board to send the control command to the controller. The controller sampling time is equal to $0.2 \mathrm{~ms}$. A classical PID controller, designed with Matlab/Simulink and dSPACE ControlDesk softwares, has been implemented for the first motions of the prototype.

Then, the targeted robot performance expressed in Tab. 1
Table 6. Objective functions and constraints for the optimal solution $\mathbf{S}^{\star}$ of the IRSBot-2

\begin{tabular}{ccc|cccc}
\hline$b b_{w}$ & $m_{I R S}$ & $f_{I R S}^{1}$ & $\tau_{\max }$ & $\tau_{R M S}$ & $\delta_{t x}$ & $\delta_{t y}$ \\
\hline$[\mathrm{mm}]$ & {$[\mathrm{kg}]$} & {$[\mathrm{Hz}]$} & {$[\mathrm{Nm}]$} & {$[\mathrm{Nm}]$} & {$[\mathrm{mm}]$} & {$[\mathrm{mm}]$} \\
432.8 & 2.169 & 49 & 157.3 & 91.2 & 0.030 & 0.136 \\
\hline \hline & \multicolumn{1}{c}{$\delta_{t z}$} & $\delta_{r x}$ & $\delta_{r y}$ & $\delta_{r z}$ \\
\cline { 2 - 6 } & {$[\mathrm{mm}]$} & {$[\mathrm{deg}]$} & {$[\mathrm{deg}]$} & {$[\mathrm{deg}]$} \\
\\
\cline { 2 - 6 } & 0.016 & 0.035 & 0.043 & 0.014 \\
\hline
\end{tabular}

have been verified experimentally.

First, the repeatability of the robot was measured with a dial indicator touching a flat device mounted under the end-effector $4 \mathrm{~cm}$ below the tool center point. Measurements were made through the robot workspace as shown in Fig. 12. 36 points have been selected into the regular dextrous workspace to characterize the robot repeatability. For each point, the mobile platform produced a $5 \mathrm{~cm}$ displacement along the arrows depicted in Fig. 12(b) at low speed for ensuring the safety of the operators near the robots whose presence was necessary during these tests. For the measurements along the axes $x_{0}$ and $z_{0}$, only the motions along the black arrows are possible. For the measurements along the axis $\boldsymbol{y}_{0}$, we added measurements also along the red arrows. 


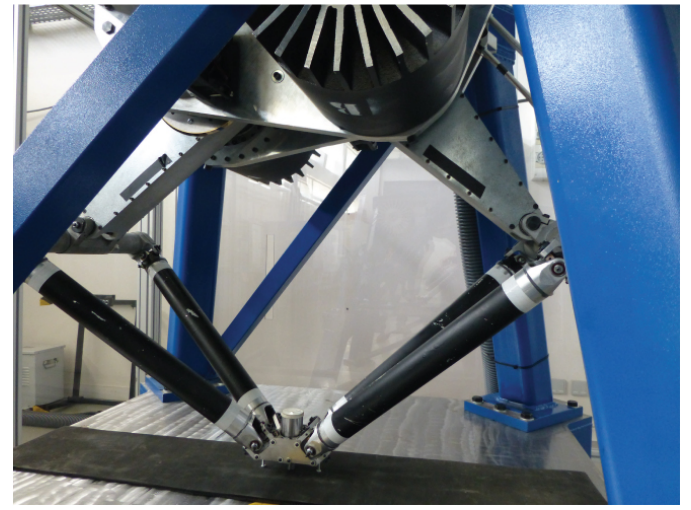

Fig. 11. IRSBot-2 robot prototype.

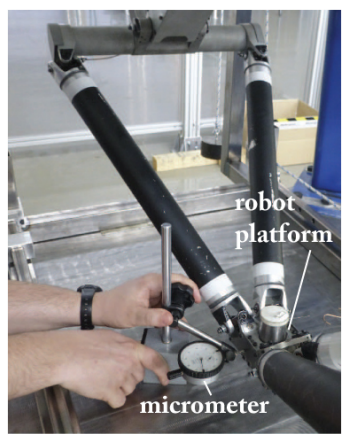

(a) Micrometer for measuring the repeatability

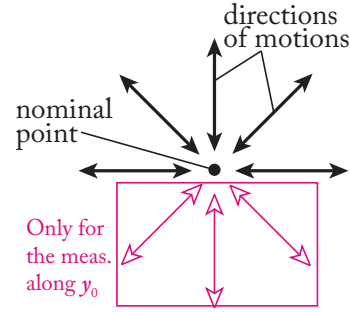

(b) Ways for approaching the point at which the repeatability is tested
Fig. 12. Benchmark for the characterization of the robot repeatability performance

Those motions have been repeated and measured three times in order to assess the robot repeatability.

The experimental results for the robot repeatability along $\boldsymbol{x}_{0}, \boldsymbol{y}_{0}$ and $z_{0}$ axes are represented in Fig. 13. It is apparent that robot repeatability is better at the bottom of the workspace. The robot repeatability is lower than 30 micrometers within the workspace. The robot repeatability along $\boldsymbol{x}_{0}$ and $z_{0}$ axes is better than the repeatability along $\boldsymbol{y}_{0}$ axis. It should be noted that point-displacement errors of the mobile platform along $\boldsymbol{y}_{0}$ axis cannot be compensated as the mobile platform motion can only be controlled in the $\boldsymbol{x}_{0}-z_{0}$ plane.

Besides, the elastostatic performance of the IRSBot-2 was analyzed by measuring the deflection of its mobile platform for a $20 \mathrm{~N}$ external force applied on the latter along axis $\boldsymbol{y}_{0}$ in order to characterize the robot performance in terms of stiffness along the axis normal to the plane of motion. The deflection of the mobile platform was measured at 90 discrete points within the robot regular dextrous workspace. The experimental setup is shown in Fig. 14 and the results are presented in Fig. 15. It turns out that the deflection of the mobile platform is lower than 120 microns through the manipulator regular workspace for a $20 \mathrm{~N}$ external force along axis $\boldsymbol{y}_{0}$.

The robot natural frequency was also measured in the home configuration $x=0 \mathrm{~m}, z=-0.54 \mathrm{~m}$ (Fig. 16). The ap-
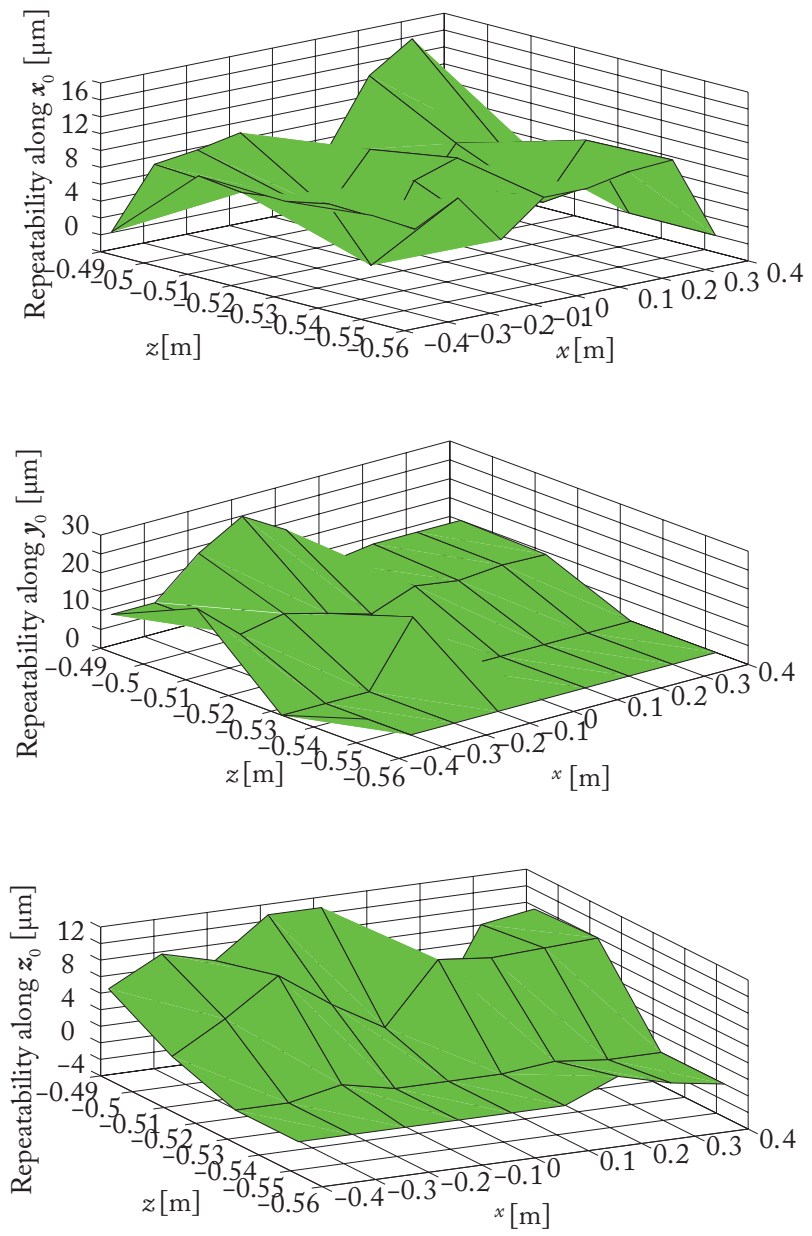

Fig. 13. IRSBot-2 prototype repeatability.

plication of experimental modal testing to the IRSBot-2 was done through impact hammer excitation, a 3D accelerometer response and data postprocessing, conducted using the DataBox software developed at LS2N and sold by MITIS company. The points and directions of excitation were chosen in order to get the maximal number of resonance frequencies. Piezoelectric triaxial accelerometers with a sensitivity of $1000 \mathrm{mV} / \mathrm{g}$ were used to get the three acceleration responses. Each measurement resolution is equal to $1 \mathrm{~Hz}$ as the acquisition time and sampling time are equal to $1 \mathrm{~s}$ and $40 \mu \mathrm{s}$, respectively.

The resonance frequencies were obtained with a fast Fourier transform of the signals given by the triaxial accelerometer. As a result, the measured resonance frequencies between 0 and $50 \mathrm{~Hz}$ are given in Table 7. The IRSBot2 natural frequencies amount to those resonance frequencies as the damping is supposed to be negligible.

Finally, the acceleration performance was characterized. The first PID controller led to large tracking errors even at relatively small accelerations (4 G, see Fig. 17). Therefore, a Computed Torque Controller (CTC) was implemented [25] in order to follow the test trajectory (Adept cycle) provided in Appendix with a maximal acceleration equal to $20 \mathrm{G}$ (Fig. 20). Results in terms of trajectory tracking and input 


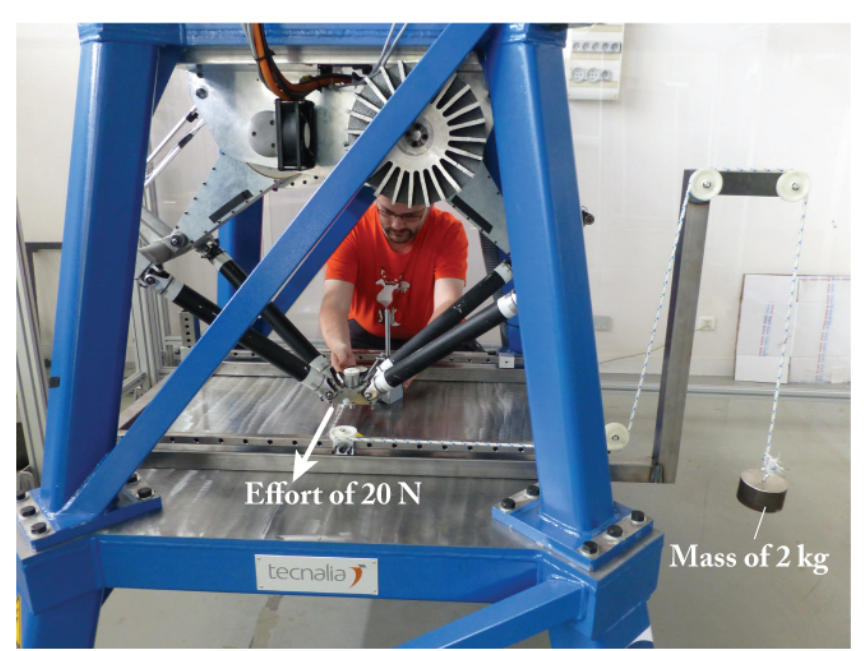

Fig. 14. Experimental setup used to characterize the elastostatic performance of the IRSBot-2.

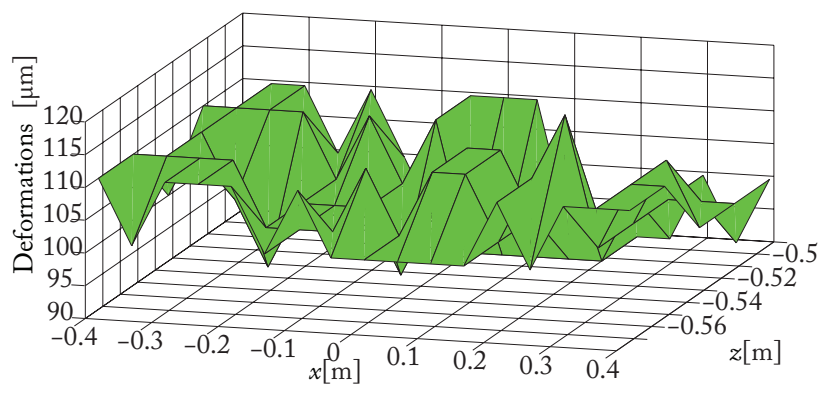

Fig. 15. Point-displacement of the IRSBot-2 mobile platform through its regular workspace for a $20 \mathrm{~N}$ external force along axis $\boldsymbol{y}_{0}$.

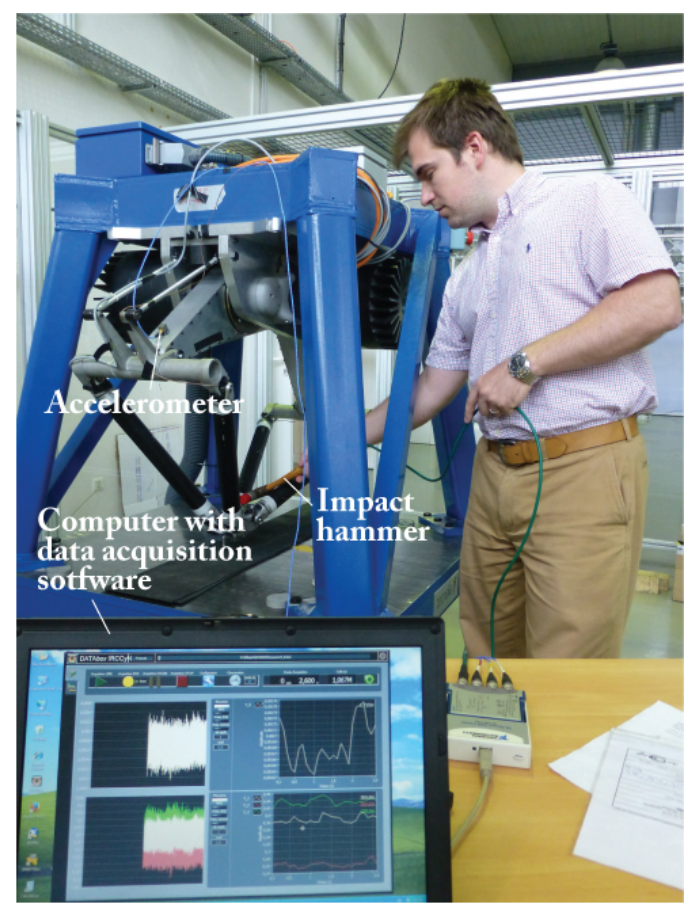

Fig. 16. Experimental setup for the robot natural frequency measurements.
Table 7. First natural frequencies measured at mobile-platform pose $(x=0 \mathrm{~m}, z=-0.54 \mathrm{~m})$.

\begin{tabular}{cc}
\hline \hline Frequency & Type of displacement \\
\hline $40 \pm 1 \mathrm{~Hz}$ & Perpendicular to the plane of mobile-platform motion \\
$40 \pm 1 \mathrm{~Hz}$ & In the plane of mobile-platform motion \\
$48 \pm 1 \mathrm{~Hz}$ & Perpendicular to the plane of mobile-platform motion \\
\hline \hline
\end{tabular}

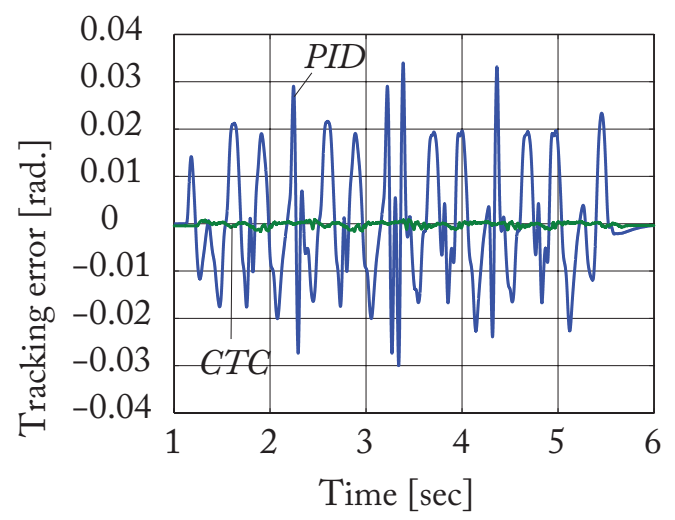

(a) Motor 1

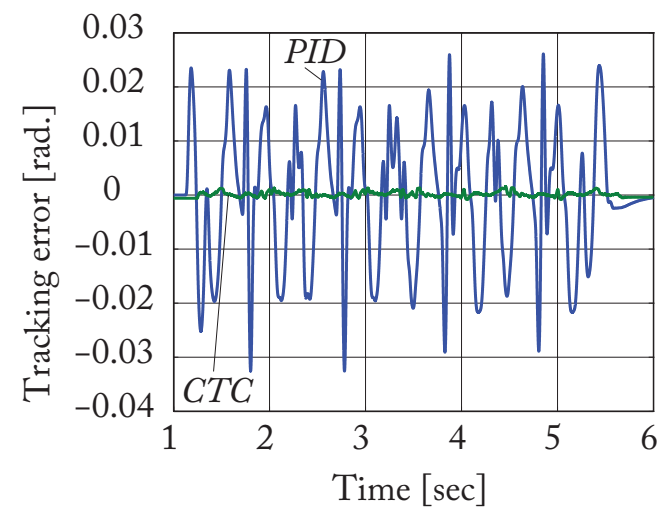

(b) Motor 2

Fig. 17. Tracking errors for the IRSBot-2 along a desired trajectory with a maximal acceleration of $4 \mathrm{G}$ and a maximal velocity of $4 \mathrm{~m} / \mathrm{s}$ between a PID controller and a Computed Torque Controller (CTC): the tracking error was divided by 20 by using the CTC. For a fair comparison, the two controllers have been set to have the same cutting frequency $(29 \mathrm{~Hz})$.

torques are shown in Fig. 18. It turns out that the controller works well because the tracking error is smaller than $10 \mathrm{mrad}$ and actuator torques remain lower than the maximal motor torque given in Table 2. The root-mean-square value of the motor torques during this motion is about $140 \mathrm{Nm}$.

At the end of the motions, oscillations at around $45 \mathrm{~Hz}$ can be observed. Those oscillations are due to the high dynamics effects that cannot be compensated with the actual CTC whose bandwith is set at $29 \mathrm{~Hz}$. 


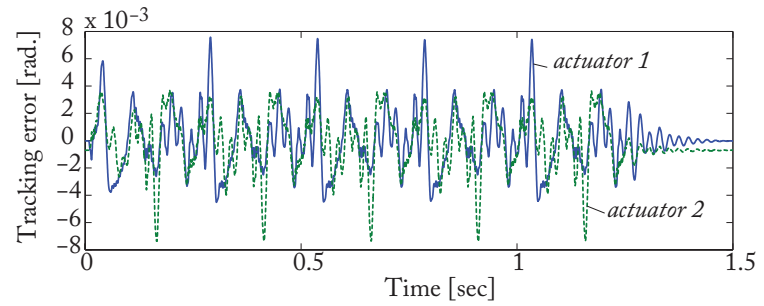

(a) Tracking errors

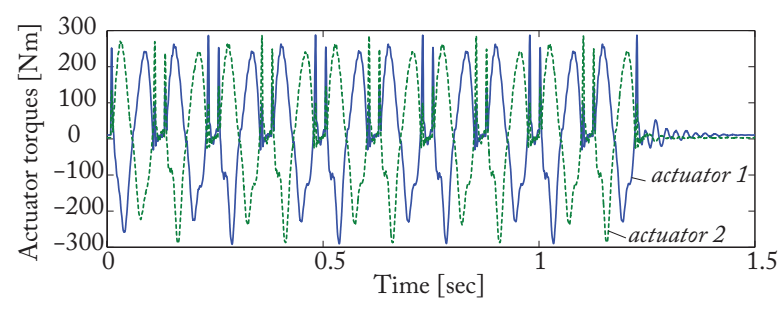

(b) Actuator torques

Fig. 18. Tracking errors and actuator torques for the IRSBot-2 along the test trajectory with desired maximal acceleration of $20 \mathrm{G}$ (see Appendix). The test trajectory is performed five times for a total time of $1.25 \mathrm{sec}$.

\subsection{Discussion}

Experimental results showed that the specifications defined at the beginning of ANR ARROW project and expressed in Tab. 1 have been met. Nevertheless, there is still some work to be done in order to improve the IRSBot- 2 performance as explained hereafter.

- Improvement of the robot absolute accuracy: in order to perform accurate pick-and-place tasks, low repeatability is not enough. Absolute accuracy must be improved. In the future, we are going to try two different approches to improve robot absolute accuracy. The first one is simple: we will record the end-effector real position by using a laser-tracker in order to quantify the error with respect to the desired configuration, and then either use this information to perform a geometric calibration [26] or to directly correct the desired position in the controller by knowing the real mobile-platform position and using error-compensation techniques [27]. However, these approaches are known not to be robust to external disturbance such as different loading on the robot. Therefore, some sensor-based controllers [28-30] will be used in the second approach.

- Design of an adaptive controller: in order to improve the trajectory tracking performance at high-speed, we intend to develop an adaptive controller [25,31] that will adapt the parameters of the dynamic model so that the tracking error is minimized.

- Vibration rejection: the IRSBot-2 robot has been designed for fast and accurate pick-and-place operations. However, the inertial phenomena involved during the high-speed motions lead to robot vibrations that decrease the robot accuracy and increase the cycle time. Future work will consider this issue by applying strate- gies for fast vibration rejections such as input shaping [32,33] and active damping [34]) techniques.

\section{Conclusion}

This paper dealt with a design procedure for a twodegree-of-freedom parallel manipulator, named IRSBot- 2 . This design procedure aimed at increasing the accuracy performance of high-speed robots. The optimal design parameters of the IRSBot- 2 were found such that the robot can reach an acceleration up to $20 \mathrm{G}$ and a $20 \mu \mathrm{m}$ multi-directional repeatability throughout its operational workspace. Besides, contrary to its counterparts, the stiffness the IRSBot- 2 should be very high along the normal to the plane of motion of its moving-platform. A semi-industrial prototype of the IRSBot- 2 has been realized based on the obtained optimum design parameters. This prototype and its main components are described in the paper. The repeatability, elasto-static performance, dynamic performance and elasto-dynamic performance of this prototype have been measured and analyzed. It turns out the IRSBot-2 has globally reached the prescribed specifications and is a good candidate to perform very fast and accurate pick-and-place operations.

\section{Acknowledgments}

This work was partially funded by the French ANR project ARROW (ANR 2011 BS3 006 01), the French Région Pays de la Loire project ARROW-Loire (Arrêté No. 2012_11768) and the joint EU Feder / "Investissements d'avenir" projects RobotEx.

The following students and engineers are dutifully acknowledged for their great help in the experiments on the IRSBot-2 robot: Francesco Allegrini, Dmitri Bondarenko, Arnaud Hamon, Guillaume Jeanneau, Philippe Lemoine, Joachim Marais, Abhilash Nayak, Angelos Platis and Victor Rosenzveig.

\section{References}

[1] Clavel, R., 1990. Device for the movement and positioning of an element in space, Dec.

[2] Caro, S., Khan, W. A., Pasini, D., and Angeles, J., 2010. "The rule-based conceptual design of the architecture of serial schönflies-motion generators". Mechanism and Machine Theory, 45(2), Feb., pp. 251-260.

[3] Angeles, J., Caro, S., Khan, W., and Morozov, A., 2006. "Kinetostatic design of an innovative schonfliesmotion generator". Proceedings of IMechE Part C: Journal of Mechanical Engineering Science, 220(7), Jan., pp. 935-943.

[4] Krut, S., Nabat, V., Company, O., and Pierrot, F., 2004. "A high-speed parallel robot for scara motions". In Proc. of 2004 IEEE International Conference in Robotics and Automation (ICRA), Vol. 4, pp. 4109 4115.

[5] Nabat, V., Pierrot, F., de la O Rodriguez Mijangos, M., Azcoita Arteche, J. M., Bueno Zabalo, R., Com- 
pany, O., and Florentino Perez De Armentia, K., 2007. High-speed parallel robot with four degrees of freedom. Patent EP 1870214 A1.

[6] Liu, X., and Kim, J., 2002. "Two novel parallel mechanisms with less than six degrees of freedom and the applications". In Proc. Workshop on Fundamental Issues and Future Reserch Directions for Parallel Mechanisms and Manipulators, pp. 172-177.

[7] Liu, X.-J., Wang, J., and Pritschow, G., 2006. "Kinematics, singularity and workspace of planar 5R symmetrical parallel mechanisms". Mechanism and Machine Theory, 44(2), p. 145169.

[8] Liu, X.-J., Wang, J., and Pritschow, G., 2006. "Performance atlases and optimum design of planar 5R symmetrical parallel mechanisms". Mechanism and Machine Theory, 41(2), pp. 119-144.

[9] Hesselbach, J., Helm, M., and Soetebier, S., 2002. "Connecting assembly modes for workspace enlargement". In Advances in Robot Kinematics.

[10] Brogardh, T., 2001. Device for relative movement of two elements.

[11] Huang, T., Li, M., Li, Z., Chetwynd, D., and Whitehouse, D., 2003. Planar parallel robot mechanism with two translational degrees of freedom. Patent WO 03055653 A1.

[12] Germain, C., Briot, S., Glazunov, V., Caro, S., and Wenger, P., 2011. "IRSBOT-2: A Novel Two-Dof Parallel Robot for High-Speed Operations". In Proc. of the ASME Design Engineering Technical Conferences.

[13] Pierrot, F., Krut, S., Company, O., Nabat, V., Baradat, C., and Saenz Fernandez, A., 2009. Two degree-offreedom parallel manipulator. Patent WO 2009/089916 A1.

[14] Germain, C., Caro, S., Briot, S., and Wenger, P., 2013. "Optimal Design of the IRSBot-2 Based on an Optimized Test Trajectory". In Proc. of ASME Design Engineering Technical Conferences.

[15] Germain, C., Caro, S., Briot, S., and Wenger, P., 2013. "Singularity-free design of the translational parallel manipulator IRSBot-2". Mechanism and Machine Theory, 64, pp. 262-285.

[16] Gosselin, C., and Angeles, J., 1990. "Singularity analysis of closed-loop kinematic chains". IEEE Transactions on Robotics and Automation, 6(3), pp. 281-290.

[17] Zlatanov, D., Bonev, I., and Gosselin, C., 2002. "Constraint singularities of parallel mechanisms". In Proceedings of the IEEE International Conference on Robotics and Automation (ICRA 2002).

[18] Briot, S., Pashkevich, A., and Chablat, D., 2010. “Optimal technology-oriented design of parallel robots for high-speed machining applications". In Proc. of the 2010 IEEE International Conference on Robotics and Automation (ICRA), pp. $1155-1161$.

[19] Merlet, J., 2006. Parallel Robots, 2nd ed. Springer.

[20] Briot, S., Glazunov, V., and Arakelian, V., 2013. "Investigation on the effort transmission in planar parallel manipulators". ASME Journal of Mechanisms and Robotics, 5(1).
[21] Germain, C., 2013. "Conception d'un robot parallèle à deux degrés de liberté pour des opérations de prise et de dépose". PhD thesis, Ecole Centrale Nantes, FR.

[22] Germain, C., Briot, S., Caro, S., and Wenger, P., in press.. "An efficient method for the natural frequency computation of parallel robots". Multibody System Dynamics.

[23] Deb, K., Agrawal, S., Pratab, A., and Meyarivan, T., 2000. "A fast and elitist multi-objective genetic algorithm: NSGA-II". In Proc. of the Parallel Problem Solving from Nature $\{\mathrm{VI}\}$ Conference, M. Schoenauer, K. Deb, G. Rudolph, Y. Xin, E. Lutton, J. J. Merelo, and S. Hans-Paul, eds., Springer. Lecture Notes in Computer Science No. 1917, pp. 849-858.

[24] Germain, C., Briot, S., Caro, S., Izard, J., and Baradat, C., 2014. "Task-oriented design of a high-speed parallel robot for pick-and-place operations". In Proceedings of the Task-based Optimal Design of Robots (ICRA 2014 WS).

[25] Khalil, W., and Dombre, E., 2002. Modeling, Identification and Control of Robots. Hermes Penton London.

[26] Hollerbach, J., Khalil, W., and Gautier, M., 2008. Handbook of Robotics. Springer, ch. 14: Model Identification, pp. 321-344.

[27] Wu, Y., Klimchik, A., Caro, S., Furet, B., and Pashkevich, A., 2015. "Geometric calibration of industrial robots using enhanced partial pose measurements". Robotics and Computer Integrated Manufacturing, 35, p. 151168.

[28] Chaumette, F., and Huchinson, S., 2008. Handbook of robotics. Springer, ch. 24: Visual Servoing and Visual Tracking.

[29] Chaumette, F., and Hutchinson, S., 2006. "Visual servo control part i: Basic approaches". IEEE Robotics and Automation Magazine, 13(4), pp. 82-90.

[30] Chaumette, F., and Hutchinson, S., 2007. "Visual servo control, part ii: Advanced approaches". IEEE Robotics and Automation Magazine, 14(1), pp. 109-118.

[31] Honegger, M., Codourey, A., and Burdet, E., 1997. "Adaptive control of the hexaglide, a 6dof parallel manipulator". In Proceedings of the IEEE ICRA, p. 543548.

[32] Singer, N. C., and Seering, W. P., 1988. Preshaping Command Inputs to Reduce System Vibration. Massachusetts Institute of Technology Artificial Intelligence Laboratory, January. A.I. Memo No. 1027.

[33] Singhose, W. E., Singer, N. C., and Seering, W. P., 1994. "Design and implementation of time-optimal negative input shapers". International Mechanical Engineering Congress and Exposition.

[34] Douat, L., Queinnec, I., Garcia, G., Michelin, M., and Pierrot, F., 2011. "Hinfiny control applied to the vibration minimization of the parallel robot Par2". In IEEE Multiconference on Systems and Control (MSC 2011). 


\section{Appendix: Definition of the optimal test trajectory}

In order to simplify the problem, polynomial motion profiles are used to find the optimal test trajectory. This trajectory must minimize the cycle time while constraining the maximum acceleration of the moving-platform of the IRSBot- 2 to be lower than $20 \mathrm{G}$ all along the path. This trajectory is computed based on an optimization procedure.

As given in Table 1, the robot should achieve the test path within $200 \mathrm{~ms}$.

The test path is depicted in Fig. 19. It is made of:

(a) a vertical portion from point $A$ to point $B$ of length $h^{\prime}$;

(b) a curve $B D$, which is symmetrical with respect to the axis passing through point $C$ and of direction $z_{0} . C$ is the mid-point of the path;

(c) a vertical portion from point $D$ to point $E$ of length $h^{\prime}$.

Let the variables $t_{0}, t_{1}, t_{2}, t_{3}$ and $t_{4}$ be the travelling time at points $A, B, C, D$ and $E$, respectively. As $A$ is the trajectory starting point and as the trajectory is symmetrical, $t_{0}=0 \mathrm{~s}$, $t_{2}=t_{4} / 2$ and $t_{3}=t_{4}-t_{1} . z_{A}$ is the coordinate of point $A$ along $z_{0}$.

The trajectory is defined in the $\left(\boldsymbol{x}_{0} O z_{0}\right)$ plane with timeparametric piecewise-polynomials, i.e., $x(t)$ and $z(t)$. Each polynomial function is of degree 5 so that the acceleration profile can be continuous with respect to time. Consequently, $x(t)$ and $z(t)$ are given by:

$$
\begin{aligned}
& z(t)= \begin{cases}z_{1}(t)=h^{\prime} s_{1}(t)+z_{A}, & \text { if } t \in\left[t_{0}, t_{1}[\right. \\
z_{2}(t)=\left(h-h^{\prime}\right) s_{2}(t)+h^{\prime}+z_{A}, & \text { if } t \in\left[t_{1}, t_{2}[\right. \\
z_{3}(t)=-\left(h-h^{\prime}\right) s_{3}(t)+h+z_{A}, & \text { if } t \in\left[t_{2}, t_{3}[\right. \\
z_{4}(t)=-h^{\prime} s_{4}(t)+h^{\prime}+z_{A}, & \text { if } t \in\left[t_{3}, t_{4}\right]\end{cases} \\
& x(t)= \begin{cases}x_{1}(t)=w / 2, & \text { if } t \in\left[t_{0}, t_{1}[\right. \\
x_{2}(t)=-w s_{5}(t)+w / 2, & \text { if } t \in\left[t_{1}, t_{3}[\right. \\
x_{3}(t)=-w / 2, & \text { if } t \in\left[t_{3}, t_{4}\right]\end{cases}
\end{aligned}
$$

where $s_{k}(t)=a_{k} t^{5}+b_{k} t^{4}+c_{k} t^{3}+d_{k} t^{2}+e_{k} t+f_{k}$ with $k=$ $1, \ldots, 5$. The boundary conditions are defined as follows:

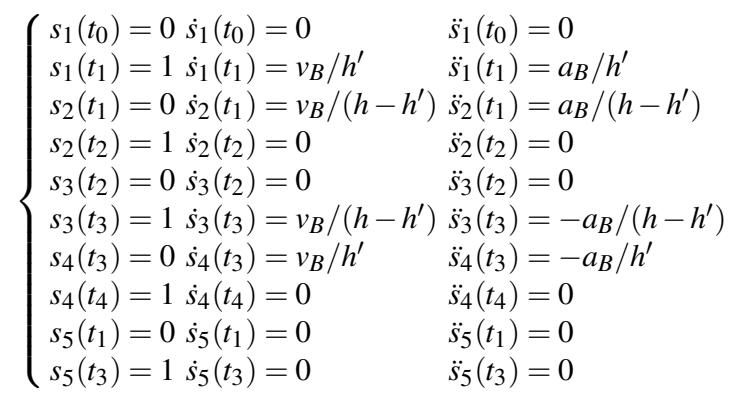

where $v_{B}$ and $a_{B}$ are the velocity and acceleration of the moving-platform at point $B$, respectively.

For given $t_{4}, t_{1}, h^{\prime}, v_{B}$, and $a_{B}$ values, Eq. (7) lead to a system of 30 linear equations with the 30 unknowns $a_{k}, b_{k}$, $c_{k}, d_{k}, e_{k}, f_{k}, k=1, \ldots, 5$ that can be easily solved.

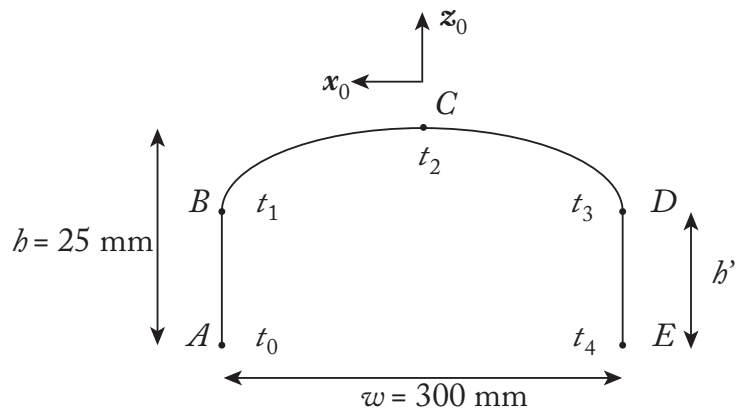

Fig. 19. Path adopted for the manipulator design.

Table 8. Optimum decision variables of $\mathrm{Pb}$. (8).

\begin{tabular}{ccccc}
\hline \hline$t_{4}[\mathrm{~s}]$ & $t_{1}[\mathrm{~s}]$ & $h^{\prime}[\mathrm{mm}]$ & $v_{B}\left[\mathrm{~m} . \mathrm{s}^{-1}\right]$ & $a_{B}\left[\mathrm{~m} . \mathrm{s}^{-2}\right]$ \\
\hline 0.1041 & 0.0055 & 2 & 0.6205 & 4.5313 \\
\hline \hline
\end{tabular}

In order to find the test trajectory, the following optimization problem is solved:

$$
\begin{aligned}
& \text { minimize } t_{4} \\
& \text { over } \mathbf{x}=\left[\begin{array}{lllll}
t_{4} & t_{1} & h^{\prime} & v_{B} & a_{B}
\end{array}\right] \\
& \text { subject to } \max \sqrt{\ddot{x}^{2}(t)+\ddot{z}^{2}(t)} \leq 20 G \quad \forall t \in\left[t_{0}, t_{4}\right] \\
& t_{1}<t_{4} / 2 \\
& 2 \mathrm{~mm} \leq h^{\prime} \leq h
\end{aligned}
$$

Problem (8) was solved with fmincon MATLAB function with multiple starting points. The optimum decision variables of problem (8) are given in Tab. 8.

Figure 20 depicts the obtained test trajectory, its velocity and acceleration profiles. It should be noted that the maximal velocity along the trajectory is equal to $6 \mathrm{~m} / \mathrm{s}$. 

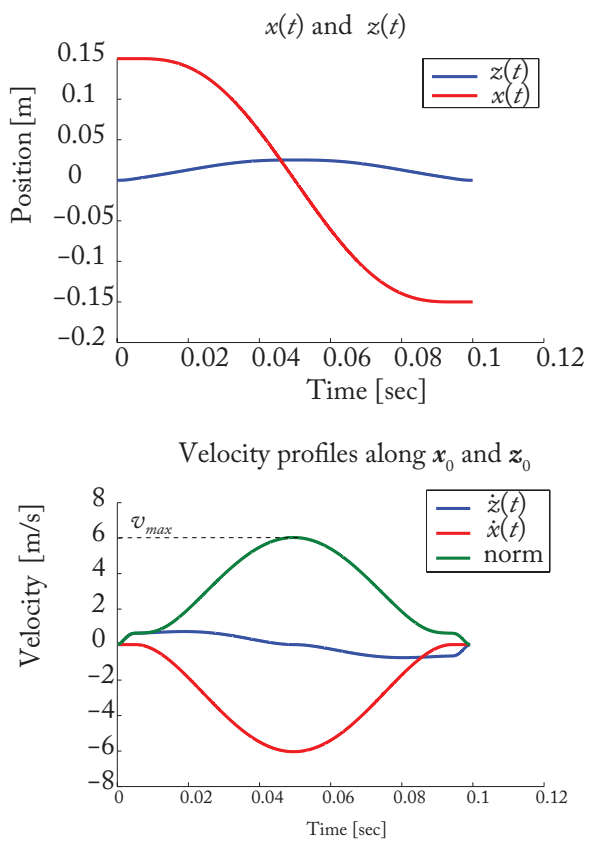

Acceleration profiles along $\boldsymbol{x}_{0}$ and $\boldsymbol{z}_{0}$

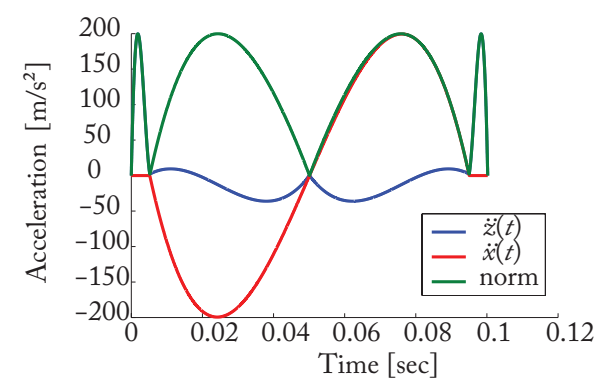

Fig. 20. Optimal trajectory and its velocity and acceleration profiles. 